\title{
Nutritional Deficiency and Wallerian Degeneration in the Rat
}

\author{
2. Effect of Thiamine Deficiency on the Concentration of Nucleic Acid and \\ Phospholipid in Intact and Sectioned Nerves \\ By W. A. MANNELL AND R. J. ROSSITER \\ Department of Biochemistry, University of Western Ontario, London, Canada
}

(Received 6 fuly 1953)

In the preceding paper (Mannell \& Rossiter, I954a) we showed that the concentration of nucleic acid in the sciatic nerve of the rat was a characteristic of the age of the animal (and not of the body-weight), whereas the concentration of phospholipid was a characteristic of the weight (and not of the age). Previously it had been shown that the increase in the concentration of nucleic acid and the decrease in the concentration of phospholipid that takes place after nerve section is more rapid in nerves from younger, and hence smaller, animals (Mannell \& Rossiter, I952, 1954b). These changes are a characteristic of the age of the animal and not of the body-weight.

In the experiments described in the preceding paper, differences in body-weight were produced by supplying certain of the animals either with unrestricted quantities of a diet deficient in protein, or with restricted quantities of a diet containing adequate protein. In the latter instance, the daily intake of food was controlled so that the mean body-weight of the animals was maintained at a level similar to that of the animals receiving unlimited amounts of the diet deficient in protein. The results achieved were considered of sufficient nutritional and general biological interest to warrant a study of a completely different type of nutritional deficiency, namely a deficiency of thiamine.

Preliminary experiments showed that in thiamine deficiency the general picture was similar to that obtained with a diet deficient in protein, or deficient in total calories. For each type of deficiency, the concentration of nucleic acid in the intact sciatic nerve was related to the age of the animal, and the concentration of phospholipid was related to the body-weight. But with thiamine deficiency there was one notable difference. The decrease in the concentration of phospholipid that occurs in the degenerating peripheral segment after nerve section, which in protein depletion and total calorie deficiency was a function of the age of the animal and not of the body-weight, took place much more slowly than would be anticipated from the age of the animal.

In this paper are described further experiments on the effect of supplying a diet deficient in thiamine on the concentration of nucleic acid and phospholipid in the sciatic nerve of the rat and also the effect of the thiamine deficiency on the changes that occur in the concentration of these substances during Wallerian degeneration. A preliminary report of these experiments has already appeared (Mannell \& Rossiter, I953). 
METHODS

Animals. The animals used were all male rats of the Sprague-Dawley strain.

Experimental plan. The general plan of the experiment was similar to that of previous experiments with protein-depleted rats (Mannell \& Rossiter, r954a). One group of animals was maintained for 24 days on a diet deficient in thiamine. The thiaminedeficient animals were compared with 'age controls', 'weight controls' and 'weight and age controls', i.e. animals receiving a diet containing adequate thiamine, but

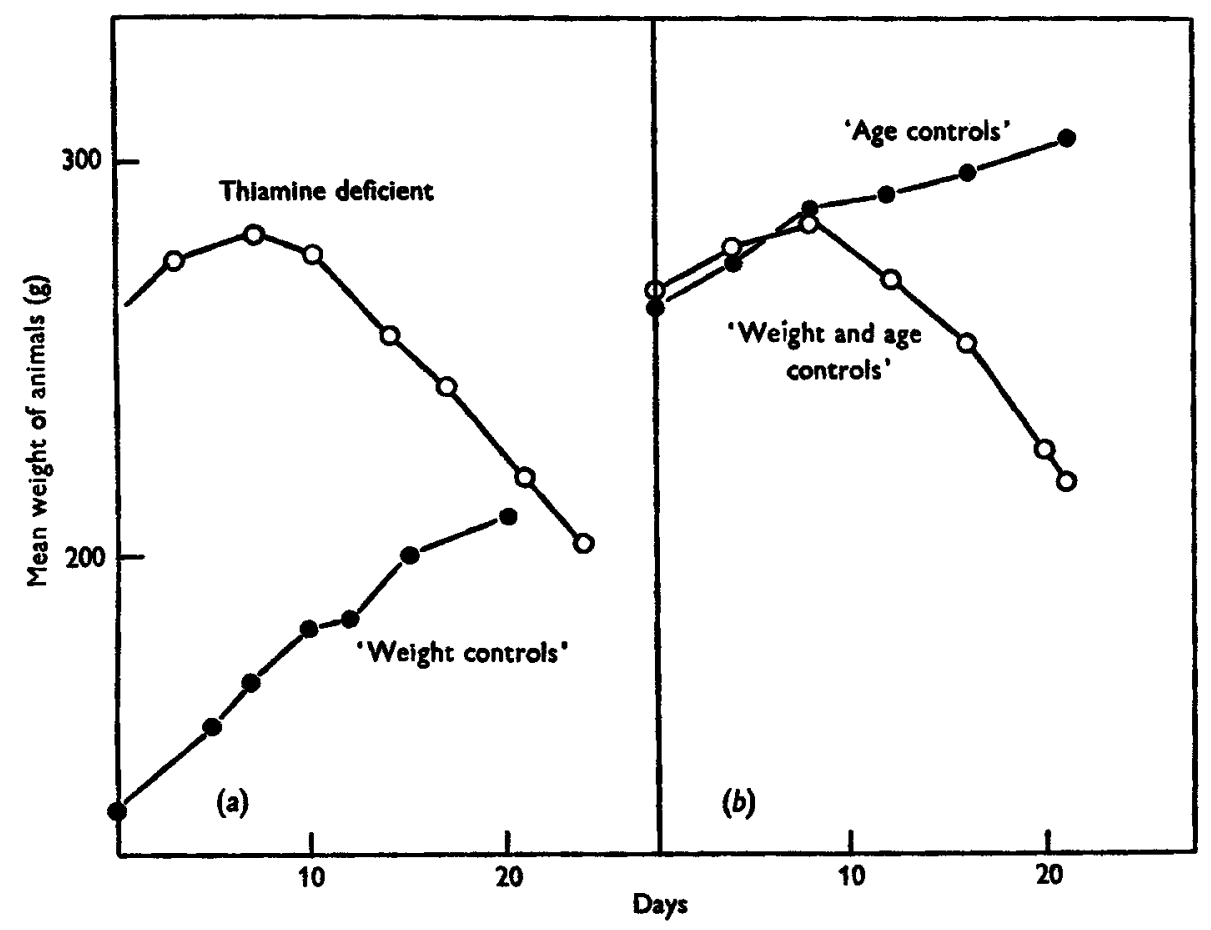

Fig. 1. Mean body-weight of rats used in first part $(a)$ and second part $(b)$ of experiment.

restricted in quantity so that the mean body-weight of the group followed that of the thiamine-deficient animals. The animals of all the control groups received a diet containing adequate thiamine. Details of the diets are given in Table $\mathrm{r}$. The mean weight changes of all four groups of animals are shown in Fig. 1.

The operative technique, analytical methods and method of recording results were the same as those described in the previous paper (Mannell \& Rossiter, 1954a).

\section{RESULTS}

\section{Intact nerves}

Nerve weight. Fig: 2 shows the significant $(P<0.001)$ correlation between the weight of the animal and the weight of the sciatic nerve for the control rats receiving unrestricted quantities of the diet containing adequate thiamine. The figure shows the 
Table I. Percentage composition of diet

$\begin{array}{lclc}\text { Vitamin mixture* } & \text { I } & \text { Maize oil } & 3.9 \\ \text { Salt mixture } & 4 & \text { Sucrose } & 68 \\ \text { Vitamin-free casein } & 20 & \text { Water } & 3 \\ \text { Cod-liver oil } \dagger & 0.1 & & \end{array}$

* Vitamin mixture:

Cod-liver oil $\dagger \quad 0.1$

$\begin{array}{lrlc}\text { Thiamine (mg) } & 50 & \text { Inositol (mg) } & 250 \\ \text { Riboflavin (mg) } & 25 & \text { p-Aminobenzoic acid (mg) } & 125 \\ \text { Pyridoxin (mg) } & 20 & \text { Biotin (mg) } & \text { I } \\ \text { Pantothenic acid (mg) } & 100 & \text { Choline (g) } & 7 \cdot 5 \\ \text { Nicotinic acid (mg) } & 100 & \text { Powdered sugar to (g) } & 100 \\ \text { Folic acid (mg) } & 25 & & \end{array}$

$\dagger$ As detailed in Table $\mathrm{I}$ in Mannell \& Rossiter (1954a). The diet deficient in thiamine was the same as the above with the exception that thiamine was omitted from the vitamin mixture.

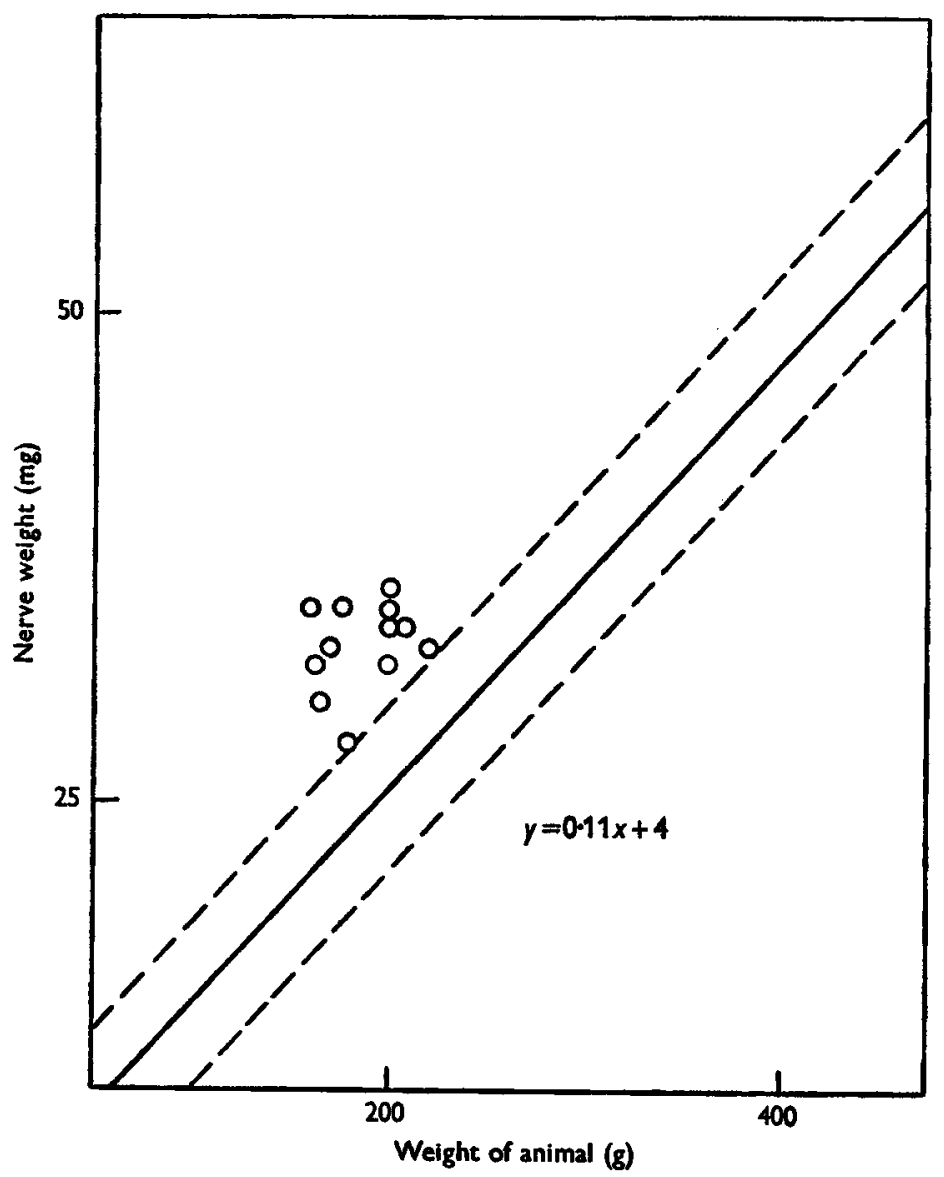

Fig. 2. Relation between the weight of the sciatic nerve and body-weight of rats maintained on an adequate diet. The solid line represents the calculated regression line and the broken lines show the standard error of the estimate of nerve weight from body-weight. Open circles (O), thiaminedeficient rats. 
calculated regression line $(y=0 \cdot 11 x+4)$ and the standard error of the estimate of nerve weight from body-weight. In the same figure are shown points for the thiaminedeficient animals. For a given animal weight, the weight of the nerve from the thiamine-deficient animals was significantly $(P<0.001)$ greater than that from control animals receiving the diet containing adequate thiamine. Protein depletion and deprivation of total calories produced similar changes (Mannell \& Rossiter, 1954a).
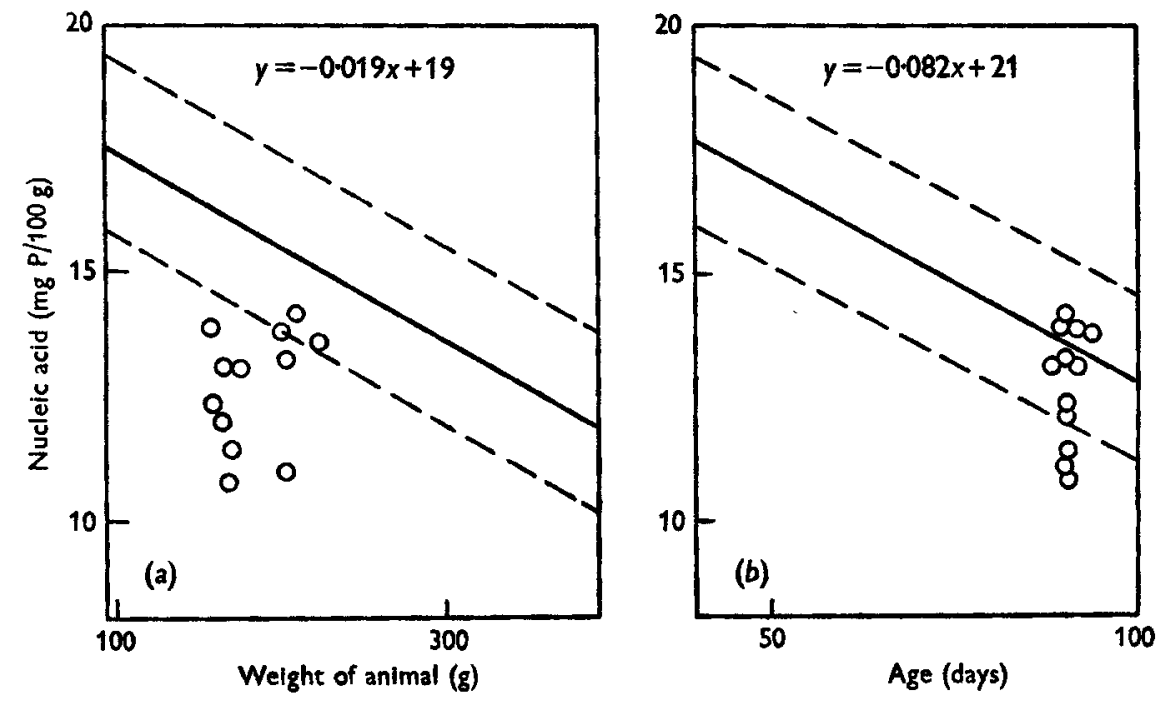

Fig. 3. Relation between the concentration of total nucleic acid in the sciatic nerve and either the bodyweight $(a)$ or the age $(b)$ of rats maintained on an adequate diet. The solid lines represent the calculated regression lines and the broken lines show the standard error of the estimate of nucleicacid concentration from either body-weight or from age. Open circles $(O)$, thiamine-deficient rats.

Nucleic acid. The unbroken lines in Fig. $3^{a}$ and $b$ show the correlation between the concentration of nucleic acid in the sciatic nerve of the rat and $(a)$ body-weight and $(b)$ age, for all of the control animals receiving unrestricted quantities of the diet containing adequate thiamine. The calculated regression equations were $y=-0.019 x+19$ for body-weight, and $y=-0.082 x+21$ for age. In the same figures are shown the standard errors of the estimate of the concentration of nucleic acid calculated from body-weight and age, respectively.

In Fig. $3 a$ and $b$ are also shown the points obtained for the thiamine-deficient animals. In Fig. $3 a$, nine of the twelve points are outside the standard error of the regression line. On the other hand, in Fig. $3 b$, nine of the twelve points are within the standard error. These results are similar to those previously reported for protein depletion and calorie deficiency, where it was concluded that age rather than bodyweight determined the concentration of nucleic acid in the sciatic nerve.

Phospholipid. Fig. $4 a$ and $b$ show similar data for phospholipid. The calculated regression lines are for the concentration of phospholipid on $(a)$ body-weight $\left(y=-0.0054^{x}+9^{.8} \mathrm{I}\right)$, and $(b)$ age $(y=-0.025 x+10.4)$ for the control animals. In the figures are also shown the points for the thiamine-deficient animals. For the body- 
weight curve (Fig. $4 a$ ), all but one of the eleven points lie within the standard error of the regression line, but for the age curve (Fig. 4 b), six of the eleven lie outside the standard error.
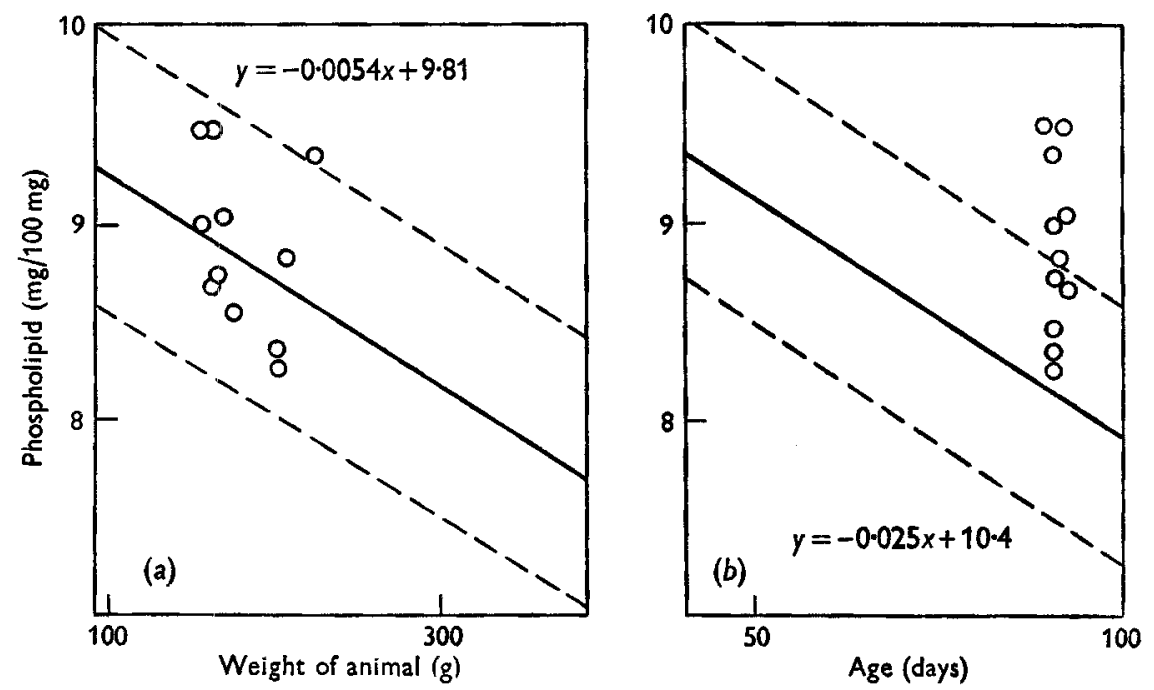

Fig. 4. Relation between the concentration of phospholipid in the sciatic nerve and either the bodyweight $(a)$ or the age $(b)$ of rats maintained on an adequate diet. The solid lines represent the calculated regression lines and the broken lines show the standard error of the estimate of phospholipid concentration from either body-weight or from age. Open circles $(O)$, thiamine-deficient rats.

Thus the findings for both nucleic acid and phospholipid are similar to those previously reported for protein depletion and for a deficient intake of total calories. Regardless of weight changes produced by each of the deficiencies, the concentration of nucleic acid is dependent upon the age of the animal and the concentration of phospholipid is dependent upon the body-weight.

\section{Wallerian degeneration}

Nucleic acid. Since the changes in the concentration of nucleic acid during the first 8 days after nerve section were also similar to those found in the previous study on protein depletion, they will not be reported in detail. The rate at which the concentration of nucleic acid increased in the nerves from the thiamine-deficient animals, or from the 'weight and age controls', receiving a restricted calorie intake, was similar to that in nerves from the 'age controls'. The increase in the younger 'weight controls' was more rapid than that in the 'age controls'.

Phospholipid. As was anticipated from the previous experiments on protein depletion, the decrease in the concentration of phospholipid in nerves from the thiaminedeficient animals was less than that found in nerves from the younger 'weight controls'. However, the decrease in the nerves from the thiamine-deficient animals was also less than that found in nerves from either the 'age controls' or the 'weight and age controls'. In thiamine deficiency the decrease in the concentration of phospholipid 
thus took place more slowly than would be expected from the age of the animals. This finding, which confirmed our preliminary experiments, is in direct contrast to the previous results for protein depletion and deficiency of total calories.

These points are illustrated in Fig. 5, which shows the relation between the decrease in the concentration of phospholipid during the first $\mathbf{8}$ days after nerve section and the concentration of nucleic acid. The figure shows the calculated regression line

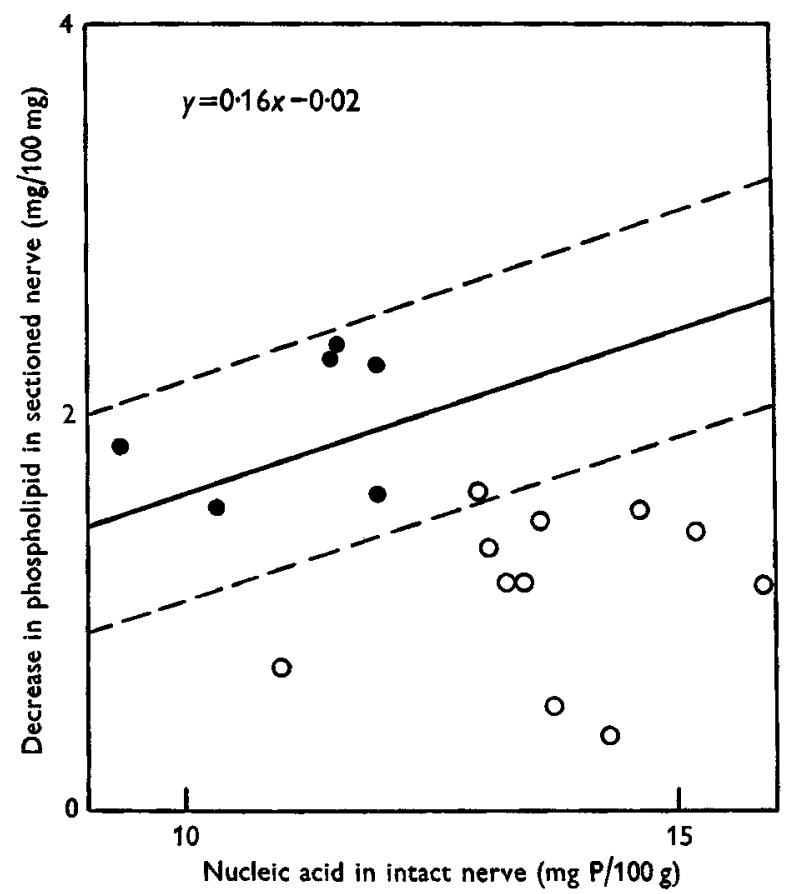

Fig. 5. Relation between the decrease in the concentration of phospholipid in the sciatic nerve of the rat during the first 8 days after nerve section and the concentration of nucleic acid in the intact nerve. The solid line represents the calculated regression line for rats maintained on an adequate diet and the broken lines show the standard error of the estimate of the decrease in the concentration of phospholipid in the degenerating nerve from the concentration of nucleic acid in the intact nerve. Open circles $(O)$, thiamine-deficient rats; closed circles $(\boldsymbol{O})$, 'weight and age controls', i.e. rats receiving a restricted calorie intake.

$(y=0.16 x-0.02)$ for all the control rats receiving unrestricted quantities of the diet containing adequate thiamine. The significant $(P<0.001)$ positive correlation between the fall in the concentration of phospholipid and the concentration of nucleic acid, which is itself correlated negatively with the age of the animal (Fig. $3^{b}$ ), confirms the previous report of Mannell \& Rossiter (1954b).

In Fig. 5 are also shown the points for the thiamine-deficient animals (open circles) and those for the 'weight and age controls' (closed circles), a group of animals that received a restricted intake of a diet containing adequate thiamine. The points for the thiamine-deficient animals, with one exception, fall below the standard error of the regression line, indicating that the decrease in the concentration of phospholipid took place more slowly than would be anticipated from the age of the animal, or from the 
concentration of nucleic acid in the sciatic nerve. In contrast, all of the points for the 'weight and age controls' fall within the standard error of the regression line. This confirmed our previous report that the decrease in the concentration of phospholipid in nerves from calorie-deficient animals was dependent upon the age of the animal, or upon the concentration of the nucleic acid in the sciatic nerve, which is correlated negatively with age (Mannell \& Rossiter, 1954a). Similar findings were reported for protein depletion.

\section{DISCUSSION}

The experiments with thiamine deficiency further support our previous conclusions that the concentration of total nucleic acid in the sciatic nerve of a rat is determined by the age of the animal, and that the concentration of phospholipid is determined by the body-weight. Previously, this generalization had been established for a wide range of ages and for a range of body-weights produced by providing a diet either deficient in protein, or containing adequate protein but deficient in total calories. Thiamine deficiency may now be added to the list of conditions that can cause a decrease in the body-weight of rats, compared with that of control animals of the same age, with no change in the concentration of nucleic acid and an increase in the concentration of phospholipid in the sciatic nerve. As was found for a deficiency of either protein or total calories, this result is different from the observations reported in the literature for other organs, such as liver, where thiamine deficiency has been shown to produce a decrease in the concentration of both pentosenucleic acid and phospholipid in terms of units of deoxyribonucleic acid (Thomson, Heagy, Hutchison \& Davidson, 1953).

The literature contains a number of reports on the effect of thiamine deficiency on degeneration in peripheral nerve. Many of the early experiments must be discounted because the deficiencies produced were usually multiple, and the changes observed could conceivably result from the deficiency of a vitamin other than thiamine. However, Lee \& Sure (1937), Davison \& Stone (1937), Swank (1940), Street, Zimmerman, Cowgill, Hoff \& Fox (194I) and Swank \& Prados (1942) described histological changes in the myelin sheath of peripheral nerves of animals subjected to a deficiency of thiamine not complicated by deficiencies of other known dietary factors. Animals studied included rats, pigeons and dogs. On the other hand, Prickett (1933), Engel \& Phillips (1938) and Wintrobe, Follis, Humphreys, Stein \& Lauritsen (1944), using rats, chickens and pigs, could find no histological changes that they believed attributable to a deficiency of thiamine. These differences were largely resolved when Prickett, Salmon \& Schrader (1939) reported that a chronic deficiency of thiamine produced more severe changes in the peripheral nervous system than an acute deficiency. In addition, Davison \& Stone (1937), Prickett et al. (1939) and Swank \& Bessey (1950) showed that a deficiency of total calories, apart from thiamine deficiency, also produced some histological changes in peripheral nerve. Our experiments show that neither in acute thiamine deficiency, nor in a deficiency of total calories, is there a degeneration of the myelin sheath in a chemical sense. There is no gross destruction of myelin and loss of myelin lipids, such as occurs in Wallerian degeneration. Changes in the fine structure of the myelin sheath cannot, of course, be precluded. In this 
regard, it is of interest to recall that Berry, Neumann \& Hinsey (1945) found that nerves from thiamine-deficient cats conducted impulses quite normally.

The finding that the decrease in the concentration of phospholipid after nerve section was slower in nerves from thiamine-deficient animals than in nerves from either the 'age controls' or the 'weight and age controls', distinguishes thiamine deficiency from protein deficiency or a deficiency of total calories.

One of the objects of this study was to test the hypothesis of Marinesco (cited by Ramon y Cajal, 1928) that proliferating Schwann cells may provide enzymes that destroy the myelin sheath in Wallerian degeneration. Since the increase in concentration of total nucleic acid in the sectioned nerves of the thiamine-deficient animals was similar to that in both the 'age controls' and 'weight and age controls', it is presumed that the cellular reaction in the peripheral segment was quite normal. However, in thiamine deficiency the decrease in the concentration of phospholipid was delayed. The experiments are thus at variance with the Marinesco hypothesis, unless it be further postulated that the enzyme systems responsible for the destruction of the myelin are themselves inhibited during thiamine deficiency.

\section{SUMMARY}

I. The concentrations of nucleic acid and phospholipid were determined in the intact and degenerating ( 8 days after nerve section) sciatic nerves of a series of rats maintained on a diet deficient in thiamine. The values were compared with those obtained for $(a)$ animals of the same age that received a diet containing adequate thiamine ('age controls'), (b) younger animals receiving the same adequate diet that were killed when they had attained a body-weight similar to that of the thiaminedeficient animals ('weight controls'), and (c) animals of the same age as the thiaminedeficient animals that were maintained on the diet containing adequate thiamine, but restricted in quantity so that their body-weight was similar to that of the thiaminedeficient animals ('weight and age controls').

2. For the animals of the control groups receiving unrestricted quantities of the adequate diet, the concentrations of both nucleic acid and phospholipid in the intact sciatic nerve were correlated negatively with either the body-weight or the age of the animal. For the thiamine-deficient animals the concentration of nucleic acid was less than that of the 'weight controls', but did not differ significantly from that of the 'age controls'. On the other hand, the concentration of phospholipid did not differ significantly from that in the 'weight controls', but was greater than that in the 'age controls'.

3. The experiments provide further evidence for the view that the concentration of nucleic acid in the intact sciatic nerve of the rat is a characteristic of the age of the animal (and not of the body-weight) and that the concentration of phospholipid is a characteristic of the body-weight (and not of the age).

4. The increase in the concentration of nucleic acid that occurs after nerve section took place more slowly in nerves from the thiamine-deficient animals than in nerves from 'weight controls', but at a rate similar to that found for the 'age controls' and the 'weight and age controls'. In contrast, although the decrease in the concentration 
of phospholipid took place more slowly than in nerves from the 'weight controls', it also took place more slowly than in nerves from either the 'age controls' or the 'weight and age controls'.

5. The experiments lend no support to the suggestion that in Wallerian degeneration proliferating Schwann cells provide enzymes that destroy the myelin sheath.

Thanks are due to Mrs D. Dickey for technical assistance. The work was supported by grants from the Defence Research Board of Canada and the National Mental Health Grants.

\section{REFERENCES}

Berry, C., Neumann, C. \& Hinsey, J. C. (I945). F. Neurophysiol. 8, 3 I5.

Davison, C. \& Stone, L. (1937). Arch. Path. 23, 207.

Engel, R. W. \& Phillips, P. H. (1938). F. Nutr. 16, 585.

Lee, J. \& Sure, B. (1937). Arch. Path. 24, 430.

Mannell, W. A. \& Rossiter, R. J. (r952). Proc. Soc. exp. Biol., N.Y., 80, 262.

Mannell, W. A. \& Rossiter, R. J. (1953). Chem. Can. 5, roo.

Mannell, W. A. \& Rossiter, R. J. (1954a). Brit. F. Nutr. 8, 44.

Mannell, W. A. \& Rossiter, R. J. (1954b). F. exp. Biol. (In the Press.)

Prickett, C. O. (1 933). Amer. F. Physiol. 107, 459.

Prickett, C. O., Salmon, W. D. \& Schrader, G. A. (1939). Amer. F. Path. 15, 25 I.

Ramon y Cajal, S. (1928). Degeneration and Regeneration of the Nervous System. London: Oxford University Press.

Street, H. R., Zimmerman, H. M., Cowgill, G. R., Hoff, H. E. \& Fox, J. C. (1941). Yale F. Biol. Med. I3, 293.

Swank, R. L. (1940). F. exp. Med. 71, 683.

Swank, R. L. \& Bessey, O. A. (1950). In Multiple Sclerosis and Demyelinating Diseases, p. 133. [H. H. Merritt, S. B. Wortis and H. W. Woltman, editors.] Baltimore: Williams and Wilkins.

Swank, R. L. \& Prados, M. (1942). Arch. Neurol. Psychiat., Chicago, 47, 97.

Thomson, R. Y., Heagy, F. C., Hutchison, W. C. \& Davidson, J. N. (1953). Biochem. F. 53, 460.

Wintrobe, M. M., Follis, R. H., Humphreys, S., Stein, H. \& Lauritsen, M. (1944). F. Nutr. $28,283$. 